\title{
What Is New in Ophthalmic Research - Focus on Ocular Surface
}

\author{
Correlation of Cell Cycle Kinetics with P63 \\ Expression in Human Limbal Epithelial Cells \\ Expanded on Intact Human Amniotic Membrane \\ E.E. Hernandez Galindo, Essen, Germany \\ Ophthalmic Res 2009;41:83-90
}

The authors analysed the expression of putative limbal stem cell marker p63 and its correlation with the cell cycle kinetics in human limbal epithelial cells (HLEC) expanded on amniotic membrane (AM) or plastic. More cells on AM than on plastic retained their BrdU label and expressed p63, indicating that AM prevents and plastic favours limbal epithelial differentiation. This reconfirms that $\mathrm{AM}$ is a suitable substrate for the cultivation of undifferentiated limbal epithelium. Nevertheless, the gradual loss of stem cells even on AM after prolonged cultivation periods suggests that transplantation should be performed at early culture stages and a need to further improve the limbal epithelial culture conditions. The BrdU-label was lost quicker than p63 expression, indicating that p63 expression was not confined to stem cells, but also in transient amplifying cells and is probably a marker for great proliferative potential, but not for stemness.

\section{Aqueous Humor Concentrations of Topically Administered Caspofungin in Rabbits}

W. Behrens-Baumann et al., Magdeburg, Germany

Ophthalmic Res 2009;41:102-105

The treatment of keratomycosis remains a clinical challenge. Caspofungin (CAS), a new echinocandin, has been developed as a systemic antimycotic drug to cover all Candida spp., the most frequent cause of fungal diseases. Due to its high molecular weight CAS is not able to penetrate the corneal epithelium. However, after corneal abrasion and serial application every $30 \mathrm{~min}$, therapeutic levels of CAS could be measured in aqueous humor. This indicates that CAS might be a promising topical drug in keratomycosis.

\author{
Immunosuppressive Property of Dried Human \\ Amniotic Membrane \\ C.Y. Park et al., Baltimore, Md., USA \\ Ophthalmic Res 2009;41:112-113
}

Human amniotic membrane is widely used to reconstruct the ocular surface in various ophthalmic diseases including corneal epithelial pathology and large defects of conjunctival tissue. The immunosuppressive activity of fresh and frozen types of human amniotic membrane have been previously reported. Dry-type human amniotic membrane ( $\mathrm{dHAM})$ is processed by dehydration using low heat and results in no live cells. The ability to fixate dHAM using biological glue is very attractive. This study demonstrated that the dHAM has the ability to suppress immune reaction despite the lack of live cells. The preservation of devitalized amniotic membrane epithelium enhanced immune suppression further compared to dHAM without epithelium. Thus, both amniotic membrane epithelium and stroma appear to be important for achieving optimal immunosuppression by amniotic membrane, regardless of its vitality.

\section{Potential Use of Riboflavin/UVA Cross-Linking in Bullous Keratopathy \\ G. Wollensak et al., Berlin, Germany \\ Ophthalmic Res 2009;41:114-117}

Collagen cross-linking of the cornea using the photosensitizer riboflavin and UVA has a protective, anti-edematous effect due to interfibrillar covalent bonds. This therapeutic principle was successfully introduced previously by the authors for the treatment of bullous keratopathy due to various causes. A marked increase of corneal transparency and visual acuity, loss of epithelial bullae and pain were achieved. Cross-linking is a new and promising perspective in the management of bullous keratopathy. In the future, it may find its place in the improvement of the symptoms of endothelial decompensation, especially in cases without prior stromal scarring.

Uwe Pleyer, Editor-in-Chief

\section{KARGER}

Fax +41613061234 E-Mail karger@karger.ch www.karger.com
(C) 2009 S. Karger AG, Base

0030-3747/09/0412-0059\$26.00/0 